\title{
ENHANCING SUPPLY CHAIN CO-ORDINATION BY MEANS OF A COLLABORATIVE PLATFORM BASED ON SERVICE ORIENTED ARCHITECTURE
}

\author{
Rubén Darío Franco, Angel Ortiz Bas, Rosa Navarro \\ Research Centre on Production Management and Engineering \\ Polytechnic University of Valencia \\ Cami de Vera $S / N$. Building $7 D$ \\ Tel. (+34) 96.387.96.80/Fax: (+34) 96.387.76.89 \\ \{dfranco| aortiz_|_ronava\}@cigip.upv.es
}

\begin{abstract}
Every new technology promises to solve a lot of problems inside and between companies and, consequently, achieve unforeseen performance improvements. Nowadays, Service-Oriented Architectures begin to be promoted as a new field where companies may realize their visions and put all their new strategies in practice.

Although they initially were focused on intra-organizational integration efforts, now they begin to be used for inter-organizational business processes engineering support in networked organizations. But, when implementing these kinds of initiatives, successful histories are mostly linked to major companies, with considerable budgets allocated to the project.

By counterpart, the INPREX project (Spanish acronym for Interoperability in Extended Processes), here presented, falls out this category. By contrast, this is an undergoing initiative leaded by a Small and Medium Enterprise (SME) and founded by a local government in Spain. In this work, we will present main project's achievements where the IDIERE Platform is used to improve the coordination in a Networked Organization scenario. Five SMEs are involved in a project which main objective is to deploy a low cost and scalable platform that allows them to improve a Collaborative Production Planning business process.
\end{abstract}

\section{MOTIVATION}

Supply Chains (SC) are a very complex type of Collaborative Networked Organization (CNO, [2, 13]) established to transform raw materials into finished products that consumers are willing to buy [16]. Players involved exchange mainly two complementary flows within this network: material and information. The flow of information is used to signal the flow of material. For example, suppliers notify that requested parts are already in transit by sending some electronic communication to the Original Equipment Manufacturer (known as Advanced Shipping Notice). 
How well managed such information flows are, along the whole supply chain, may restrict the overall performance. Poorly managed or distorted information may cause, for instance, poor inventory and production practices [1].

Several organizational models apply when dealing with this environment. Initially, Extended Enterprises [13] was used when a dominant player extended its boundaries for its operations.

In this context, it's very important to take into account that electronically supported networks, usually may present some relevant features that may affect the information sharing practices:

- Heterogeneity: Inside a SC, it's possible to find a set of legacy (or usually, proprietary) systems which are heterogeneous and not designed to be interactive. They are usually implemented using different technology, different languages and for different operating platforms.

- Scalability: Companies (or SC Players) usually must be able to integrate their new applications into the distributed system without affecting the other components of the system

- Adaptability: The interactions between players need to be changed as needed. Infrastructures and systems should be flexible and easy to accommodate to new requirements.

- Autonomy: Companies have their own IT/IS policies and must keep them besides well-established relationships. In most of cases, companies get involved in several supply chains (or clients) and additional efforts for information exchange practices are needed in each case.

In this work, we will present a case study that has been carried out under the INPREX Project (Spanish acronym for Interoperability in Extended Processes) and where a collaborative technological platform based on Service Oriented Architectures foundations has been used to enhance the co-operation when Collaborative Production Planning capabilities are required in a Supply Chain.

The paper is structured as follows: Section 2, introduces different models that historically have been used to solve this problem inside Networked Organizations; Section 3 is devoted to briefly show how Service Oriented Architectures are well positioned to automate the information sharing process and consequently reach inter-organizational business processes automation. Section 4, depicts the IDIERE platform main concepts which have been used to conduct the business case presented on Section 5. Finally, Section 6 states some conclusions of our work.

\section{SERVICE ORIENTED ARCHITECTURES FOR INFORMATION SHARING AND INTER- ORGANIZATIONAL BUSINESS PROCESS INTEGRATION}

It's argued that a Service Oriented Architecture (SOA) is many things [5]. Mainly, it can be conceived as a technological enabler or as a building block for new collaborative models.

Positioning companies under this paradigm implies not only adopting XML and web services related standards for process co-ordination [8] but also creating a new way of thinking about how information and knowledge must flow inside these breeding environments [2]. From this combined approach, it's possible to consider 
that SOA may be useful for enhancing Supply Chain co-ordination when engineering and deploying distributed business process, due these major reasons:

- The capability of closing the gap existing between the modelling and the operational phases of the business process engineering, because we are able of modelling them with Business Process Modelling Notation (BPMN), for instance, and getting one executable version of the process by using BPEL4WS.

- The emerging web-services technologies allow different levels of abstraction for business processes modelling and representation. Then, any CNO member in charge of executing some assigned activity of the process may locally explode it (preserving the public interface) by using their own BPEL4WS model as well.

- Also, always that one activity interface is preserved executors can be switched [12] depending on conditions under which process/criteria to be met.

Additionally, a complementary concept needs to be introduced here. According to Kraft (2002) web services concept must be refined by extending it in order to consider it as an object web service. An object web service has a set of methods that can be accessed by their interfaces (object oriented programming analogy).

\section{A SERVICE ORIENTED PLATFORM FOR BUSINESS PROCESS MANAGEMENT}

The INPREX project looks for innovative methods and tools for supporting whole business process life cycle. By doing so, a collaborative platform is being developed in order to achieve this goal.

Based on SOA principles, BPM and Web Service Objects the IDIERE Architecture has been defined as follows [7], in order to achieve Machine to Machine Automation of distributed business processes (see Section 2):

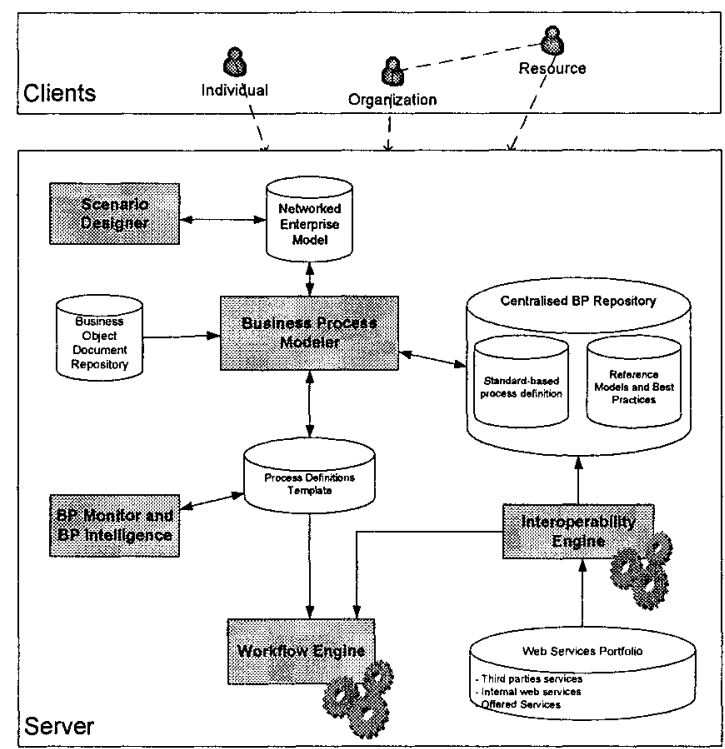

Figure 1 - The IDIERE Architecture 
Although the system supports distributed business process enactment, the IDIERE Platform isn't P2P oriented. Instead of this, it is composed of two major components: a central server which stores process definitions and orchestrate activities execution and a set of clients which assume different roles along process life cycle.

More precisely, inside the platform it's possible to identify:

- The IDIERE Server: it supports two major phases: process engineering and execution. It allows defining collaborative business processes structure, storing them in a common repository, registering executors, assigning execution method and executors to individual activities or sub processes, monitoring delegated activities, deploying a set of indicators for performance measurement.

- Client-side: in terms of users, there would be a set of nodes which may be connected to the platform in order to notify each assignment status. Each node represents the instantiation of one executor for each process instance. Correspondingly, each deployed thin client, will act as Task Manager for the system (in workflow sense) but having some extended functionality.

Distributed business process may be conceived as a set of activities which are assigned to different roles to be accomplished. When modelling this kind of processes, is not always possible to keep the same abstraction level for each activity.

In fact, it depends on how much detail can be gathered. More, initial steps in process modelling always begin with a more or less clear picture but without so much detail.

Then, we prefer to define these related concepts:

"D1: an Execution Unit is a work package that may be composed of a single activity or a whole process and that could be assigned to some executors which have the proper knowledge and capacity to accomplish the task"

From the Information Management perspective, the execution unit can be seen as a computational function ws which maps some Data Inputs into Data Outputs trough some internal logic not accessible by others (in a black box way).

This led us to the concept of service and service provider. If each execution unit is wrapped under some interface that can be located and consumed by third parties, it is possible to consider it as a service that may be provided by some service provider.

Then, service providers or executors can be defined as follows:

D2: executors are those service providers (organizations or their resources) that are capable of accomplish some execution unit for the global process by providing and consuming third-parties services.

And relaying on SOA principles, it could be convenient to add:

"...by means of web services interface definitions and a supporting

data model."

Then, executors have the following structure: 


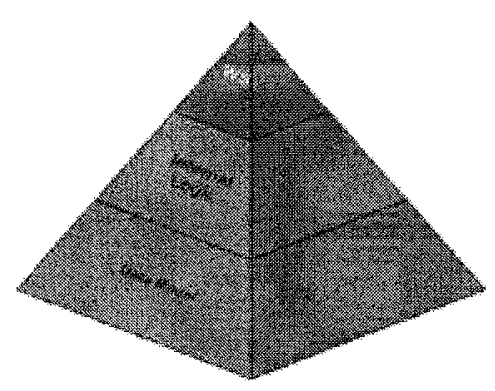

Figure 2 - Executor's architecture

Then, the IDIERE execution model is based on the orchestration of execution units' accomplishment that has been previously assigned to executors.

\section{CASE STUDY}

\subsection{Business scenario}

The SME is a first-tier supplier (design, procurement, production and hand-over), for a number of world-class manufacturers of the automobile industry.

The company's principal line of production consists of metal reinforcements for the main panels of a vast variety of cars of the above mentioned manufacturers, spare-wheel and their corresponding retainer, engine mountings, pedals, supports for the rear seats, and separate compartments, as well as other parts of the body.

Thus the SME becomes a Full Service Supplier (and high level sub-assembly supplier) for the Original Equipment Manufacturer (OEM) meaning that the company is a 1st-tier supplier that is responsible for its own logistics and independently chooses its own suppliers (2nd-tiers), the components of its products, etc.

The Supply Chain, as it is now, has a relatively low degree of synchronization and collaboration. Generally, although the production plans of the company that is directly connected with the demand are those who launch the supply chain's production, certain information that needs to be drawn upstream is held up and sequentially the cycles of planning and production decrease, and therefore the delivery time of the final item is significantly affected.

Currently, this information flow is relatively restricted. The SME receives OEM's demand planning and makes its production plans and attendant orders to 2nd-tiers according to it. But when small- scale changes and variation in demand coming from the OEM arrive, SME's production plans and order needs change, but the sub-tiers are not informed on time and thus do not have the time they may need to change their own planning, if this is possible.

This lack of collaboration (either for synchronization or visibility) results in a great number of problems arising from the uncertainty of information, whose impact is reflected throughout the supply chain. 


\subsection{Proposed solution}

The networked enterprise involved in the solution directly includes the stamping firm (SME) and four more companies in the same scenario: a subsidiary stamping plant, a painting firm, a welding subcontractor and a second welding and assembly subcontractor. We have applied the IDIERE platform principles in order to achieve a successful reengineering of the Production Planning Process (PPP).

Inside this process, three kinds of executors were identified (see Fig 3):

1. Productive: they transform some inputs (raw material or components) into out-puts (components) by performing of some activity (stamping, painting, welding or similar).

2. Transport: it moves containers between productive executors.

3. Warehouse: they stores finished or semi-finished goods

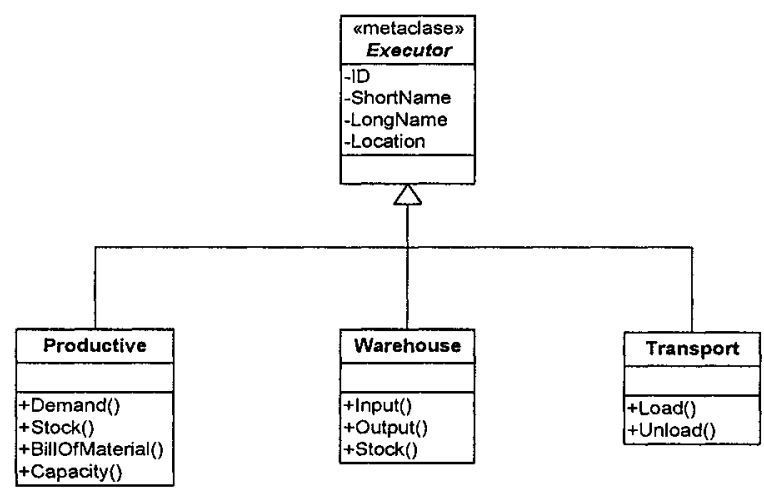

Figure 3 - Organizational resources have been modeled as executors

Once executors have been modelled, supporting services were developed for each kind of executor. Then, there is a set of services belonging to each one of them. For instance, productive executors are able of notify stocks, capacities, enact their Bill of Material, receive demand, put orders and so on.

Table 1 - Supported services (illustrative purposes only)

\begin{tabular}{|l|l|l|}
\hline Executor & ServiceName & Description \\
\hline Productive & NotifyDemand() & $\begin{array}{l}\text { It allows to know which is the demand } \\
\text { for some reference during the next days }\end{array}$ \\
\cline { 2 - 3 } & NotifyStock() & It returns stock of some reference \\
\cline { 2 - 3 } & BillOfMaterial() & $\begin{array}{l}\text { It returns those references which } \\
\text { compose other one }\end{array}$ \\
\cline { 2 - 3 } & Capacity() & $\begin{array}{l}\text { Notify agreed capacity about some } \\
\text { reference based on signed contracts }\end{array}$ \\
\hline Warehouse & Input() & Register receptions of material \\
\cline { 2 - 3 } & Output() & Register material consuptions \\
\cline { 2 - 3 } & Stock() & Notify availability \\
\hline
\end{tabular}


After that, we modelled the production process of each reference (almost ninety) as a business process within which each activity (stamping, welding, and painting) is associated with the corresponding executor and its services by means of a contract that includes references, capacities, shipping units, etc. (see Fig. 4).

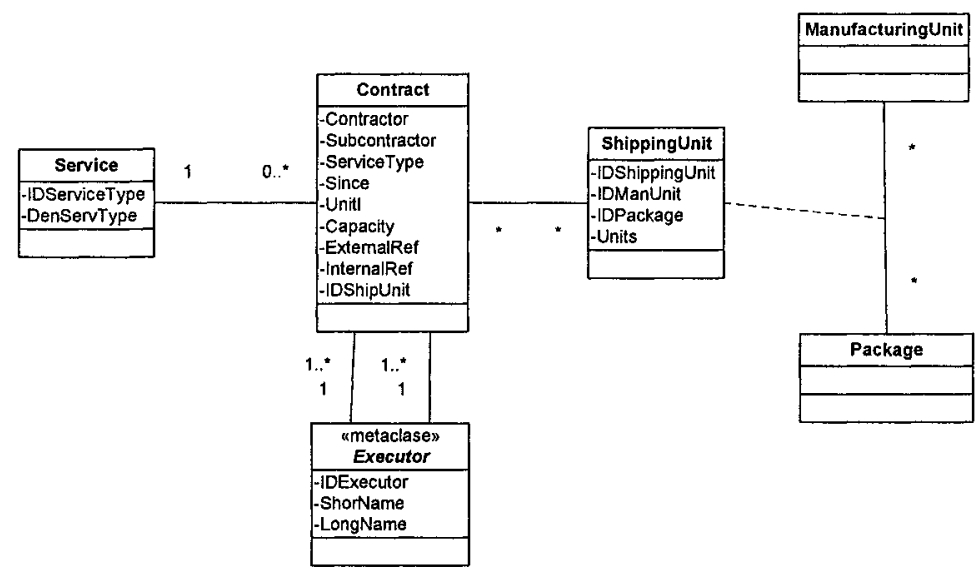

Figure 4 - Contracts establishes services to be provided between executors

Manufacturing some reference can be seen as an orchestration of those services that must be provided by all involved executors of the process. By establishing P2P contracts (or Service Level Agreements) it's possible to define the path from raw material to finished product (and consequently, the Bill of Material).

Once execution units and executors to accomplish them have been defined, an additional module that uses such capabilities and definitions has been developed: a collaborative planner which, based on process definitions and executors' services, allows checking SME's plans feasibility and needs.

\section{CONCLUSIONS}

In this work, we have tried to depict how Supply Chains are facing new challenges in reconfiguring themselves when looking for flexibility and adaptability.

The right way seems to be information sharing policies shifting by providing interoperability mechanisms that automate the information retrieval and sharing practices. By counterparts, Internet is fuelling Service Oriented Architectures deployment by supporting them not only as a new technology but also as a new way of business thinking.

We have tried to show how, by using SOA principles, manufacturing practices can be improved in a Supply Chain. We have created two building blocks, execution units and executors, which allow us to model a process as an orchestration of web services invocations.

The case presented here is a small example that shows how small and medium enterprises may also embrace this technology in order to improve the co-operation inside the network. 


\section{Acknowledgments}

The INPREX (Interoperability in Extended Processes) project is an undergoing project founded by the Spanish government by means of its Office for Science and Technology.

\section{REFERENCES}

1. Brynjolfsson, E. and Smith, M. (2000) "Frictionless Commerce? A Comparison of Internet and Conventional Retailers". Management Science, April 2000 (volume 46, number 4), pages 563-585.

2. Camarinha-Matos, L. and Afsarmanesh, H. (2001). Dynamic Virtual Organizations, or Not So Dynamic? BASYS Proceedings pp 111-124

3. Camarinha Matos, L. and Afsarmanesh, H. (2004). "The Emerging Discipline of Collaborative Networks". In Virtual Enterprises and Collaborative Networks. Kluwer Academic Publishers. PROVE'04 Proceedings

4. Clarke, R. (2004). "Open source software and open content as models for eBusiness" Presented at 17th International eCommerce Conference, Slovenia, June 2004.

5. Erl, T. (2004). Service Oriented Architecrures: A Field Guide. Prentice Hall Ed. New Jersey

6. Fingar, P. and Smith, H. (2003). Business Process Management: The third wave. Meghan-Kiffer Press, Tampa, Florida

7. Franco, D., Ortiz Bas, A. and Anaya, V. "An Architecture for Managing Distributed Business Processes in Networked Organizations". International Workshop on Enterprise Integration, Interoperability and Networking (EI2N'2005). Proceedings to be published.

8. Hagel, J, and Brown, J.S. (2002). Orchestrating Business Processes - Harnessing the value of web services technology. Working Paper available at www.johnhagel.com

9. Handfield, R. and Nichols, E. (2002). Supply Chain Redesign. Transforming Value Chains into Integrated Value Systems. Ed. Prentice Hall. New Jersey.

10. Kraft, R. (2002). A Model for Network Services on the Web. Intermational Conference on Internet Computing 2002: 536-541

11. Linthicum, D. (2004). Next generation application integration: from simple information to web services. Addison-Wesley Information Technology Series.

12. Mowshowitz, A. (1999). 'The switching principle in virtual organization', Virtual Organization Net eJOV, vol. 1 , no. 1, pp. 6-17

13. Ortiz, A., Franco R.D. y Alba, M. "V-CHAIN: Migrating from Extended to Virtual Enterprise within an Automotive Supply Chain". PROVE'03 Proceedings. Processes and Foundations for Virtual Organizations.

14. Rappa M. (2003) 'Business Models on the Web', accessed on January, 2004 at http://digitalenterprise.org/models/models.html

15. Rust, R.T. and Kannan, P.K. (2002) E-Service: New Directions in Theory and Practice. ME Sharpe, Armonk, New York.

16. Shapiro, J. (2001). Modeling Supply Chain. Duxbury Press, 2001. 